Check for updates

Cite this: Chem. Commun., 2017, 53, 11056

Received 15th July 2017,

Accepted 19th September 2017

DOI: $10.1039 / c 7 c c 05493 a$

rsc.li/chemcomm

\section{Structure and dynamics elucidation of ionic liquids using multidimensional Laplace NMR $\dagger$}

\author{
Muhammad Asadullah Javed, (D) ${ }^{a}$ Susanna Ahola, ${ }^{a}$ Pär Håkansson, (D) ${ }^{a}$ \\ Otto Mankinen, (D) ${ }^{a}$ Muhammad Kamran Aslam, ${ }^{a}$ Andrei Filippov, (DD ${ }^{\mathrm{b}}$ \\ Faiz Ullah Shah, (D) ${ }^{\mathrm{b}}$ Sergei Glavatskih, (D) ${ }^{\mathrm{cd}}$ Oleg N. Antzutkin (D) ${ }^{\mathrm{b}}$ and \\ Ville-Veikko Telkki (D)*a
}

We demonstrate the ability of multidimensional Laplace NMR (LNMR), comprising relaxation and diffusion experiments, to reveal essential information about microscopic phase structures and dynamics of ionic liquids that is not observable using conventional NMR spectroscopy or other techniques.

Ionic liquids (ILS) are salts that consist of ions and have, by definition, melting points below $100{ }^{\circ} \mathrm{C}$. They have unique physical and chemical properties such as high ionic conductivity, negligible vapour pressure, nonflammability, broad liquid phase temperature ranges, and high thermal stability, which all make ILs attractive in many scientific and technological applications. The applications include organic synthesis and catalysis, gas separation, extraction of metals, lubrication, electrochemistry, crystallization media for pharmaceutically active compounds and functional materials, etc. $^{1}$

NMR relaxation and diffusion experiments provide versatile information about the dynamics and structure of substances such as proteins, polymers, liquid crystals and porous media. ${ }^{2}$ They may also improve chemical resolution by distinguishing different components in complex systems without spectral resolution. ${ }^{3}$ The relaxation and diffusion data consist of exponentially decaying components and the processing requires a Laplace inversion in order to determine diffusion coefficient and relaxation time distributions. ${ }^{2}$ Therefore, these methods are referred to as Laplace NMR (LNMR). Like in traditional NMR spectroscopy, a multidimensional approach significantly enhances the resolution and information content of LNMR. ${ }^{4}$ The approach makes it possible

\footnotetext{
${ }^{a}$ NMR Research Unit, University of Oulu, P.O. Box 3000, 90014 Oulu, Finland. E-mail: ville-veikko.telkki@oulu.fi

${ }^{b}$ Chemistry of Interfaces, Luleå University of Technology, SE-97187 Luleå, Sweden

${ }^{c}$ System and Component Design, KTH Royal Institute of Technology, SE-10044 Stockholm, Sweden

${ }^{d}$ Soete Laboratory, Department of Electrical Energy, Systems and Automation, Ghent University, B-9000 Ghent, Belgium

$\dagger$ Electronic supplementary information (ESI) available: Additional details of experimental methods and results and relaxation data in terms of the microscopic model. See DOI: 10.1039/c7cc05493a
}

to correlate diffusion coefficients and relaxation times, and enables the investigation of chemical exchange even in the case when the exchanging sites are not resolved in the spectrum. The method requires a reliable and robust multidimensional Laplace inversion algorithm for extracting the diffusion coefficient and relaxation time distributions from the experimental data. ${ }^{5}$

NMR relaxation and diffusion experiments have been widely used to investigate physicochemical properties, hydrogen bonding, aggregation, solvation dynamics and atomic level interactions of ILs. ${ }^{6}$ However, to the best of our knowledge, the ability of Laplace inversion algorithms to provide distributions of relaxation times and diffusion coefficients has not yet exploited in studies of ILs. Furthermore, the potential of multidimensional LNMR to provide unique information about correlations and exchange is yet unexplored in the IL context.

In this work, we demonstrate that a combination of several one-dimensional (1D) and two-dimensional (2D) LNMR experiments can provide important microscopic information about the phase structures of ILs, which is not possible using conventional NMR spectroscopy or other methods. We concentrate on the investigation of a halogen-free orthoborate based ionic liquid (hf-BIL). ${ }^{7}$ The affinity to absorb water, high polarity and toxicity make halogen containing ILs undesirable in many applications, and hf-BILs provide environmentally more friendly alternatives. Tetraalkylphosphonium and tetraalkylammonium based hf-BILs are highly hydrophobic and they exhibit good lubrication properties (considerably better wear and friction reduction as compared to standard fully-formulated oil-based lubricants), low melting points and high thermal and hydrolytic stability.

Preliminary NMR studies have shown that one of the hf-BILs, consisting of the trihexyl(tetradecyl)phosphonium, $\left[\mathrm{P}_{6,6,6,14}\right]$, cation, and bis(mandelato)borate, $[\mathrm{BMB}]$, anion (see insets in Fig. 1a), ${ }^{7 a}$ has two significantly distinct diffusion coefficients $(D)$ at temperatures below $320 \mathrm{~K}$, which indicates the presence of two coexisting dynamic liquid phases. ${ }^{8}$ Variable temperature ${ }^{1} \mathrm{H}$ NMR diffusion coefficient distributions measured at $300 \mathrm{MHz}$ (under a 7.05 T magnetic field, see details in the ESI $\dagger$ ) shown in 


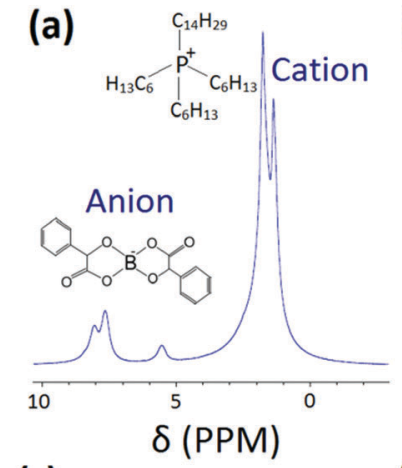

(c)
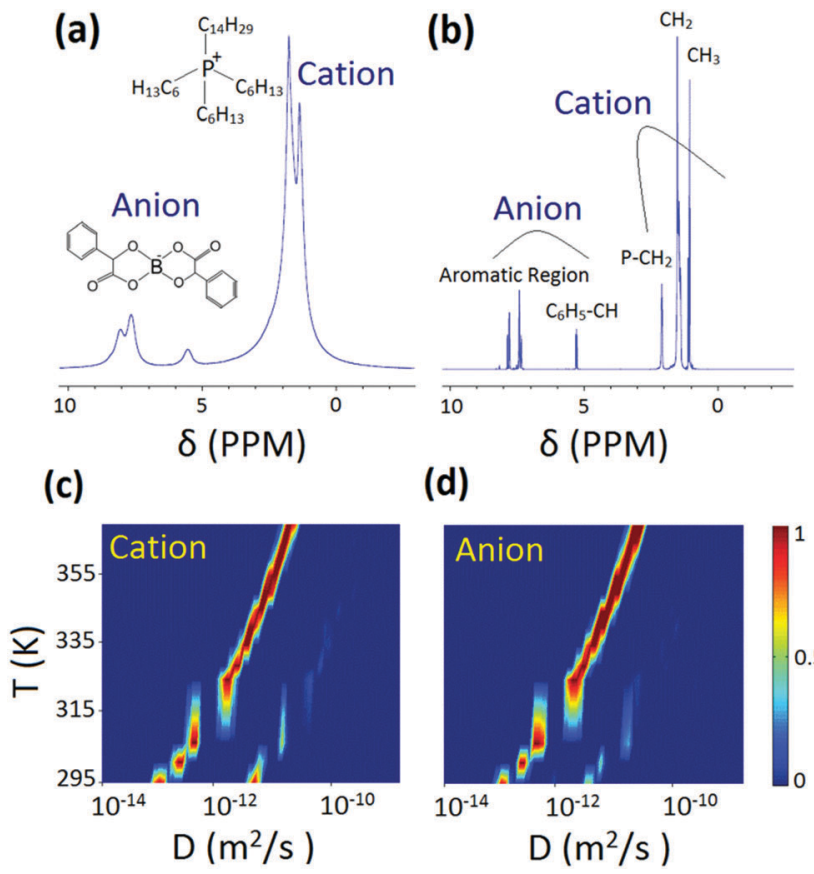

(d)

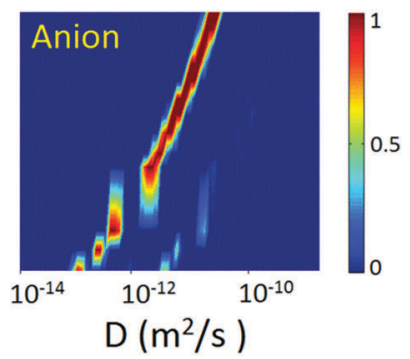

Fig. $1{ }^{1} \mathrm{H}$ NMR spectra of the $\left[\mathrm{P}_{6,6,6,14}\right][\mathrm{BMB}] \mathrm{IL}$ measured at (a) 296 and (b) $379 \mathrm{~K}$. The lines are much broader at the lower temperature due to the slower mobility of ions in the IL. Diffusion coefficient distributions of the cation (c) and the anion (d) as a function of temperature. The experimental time of the diffusion experiment was 8 hours with 8 scans (at each temperature).

Fig. 1c and d support this observation. The experiments were carried out using a pulse-field-gradient stimulated echo (PGSTE) method with bipolar gradients (BP), ${ }^{9}$ and, in the analysis, either cation resonances between 0 and 3 ppm or anion resonances between 7 and 9 ppm (see Fig. 1a and b) were integrated. Both cations and anions have two different diffusion coefficients. Laplace inversions were performed with a MATLAB based program provided by P. Callaghan. ${ }^{5,10}$ The $D$ values are almost equal for cations and anions. The $D$ value of the phase characterized by slow diffusion (later called "slow phase") is very small, about $1 \times 10^{-13} \mathrm{~m}^{2} \mathrm{~s}^{-1}$ at room temperature. The $D$ value of the other phase ("fast phase"), about $5 \times 10^{-12} \mathrm{~m}^{2} \mathrm{~s}^{-1}$, is almost two orders of magnitude larger than that of the slow phase. Based on the theoretical modelling shown below, we propose a possible hypothesis that the slow and fast "phases" arise due to aggregates formed by anions and cations and free (non-aggregated) ion pairs, respectively.

In order to find $T_{2}$ relaxation times of the slow and fast phases, we carried out a $D-T_{2}$ correlation experiment. ${ }^{11}$ The pulse sequence of the experiment, shown in Fig. 2a, includes the PGSTE-BP part for the diffusion encoding, followed by the Carr-Purcell-Meiboom-Gill (CPMG) ${ }^{12} T_{2}$ loop. The 2D correlation map resulting from the Laplace inversion of the experimental data, shown in Fig. 2b, includes two peaks corresponding to the slow and fast phases. The peaks reveal that $T_{2}$ of the slow phase, about $6 \mathrm{~ms}$, is significantly shorter than that of the fast phase, about $80 \mathrm{~ms}$. The integrals of the peaks are roughly equal, similarly to the $1 \mathrm{D}$ diffusion coefficient distribution.
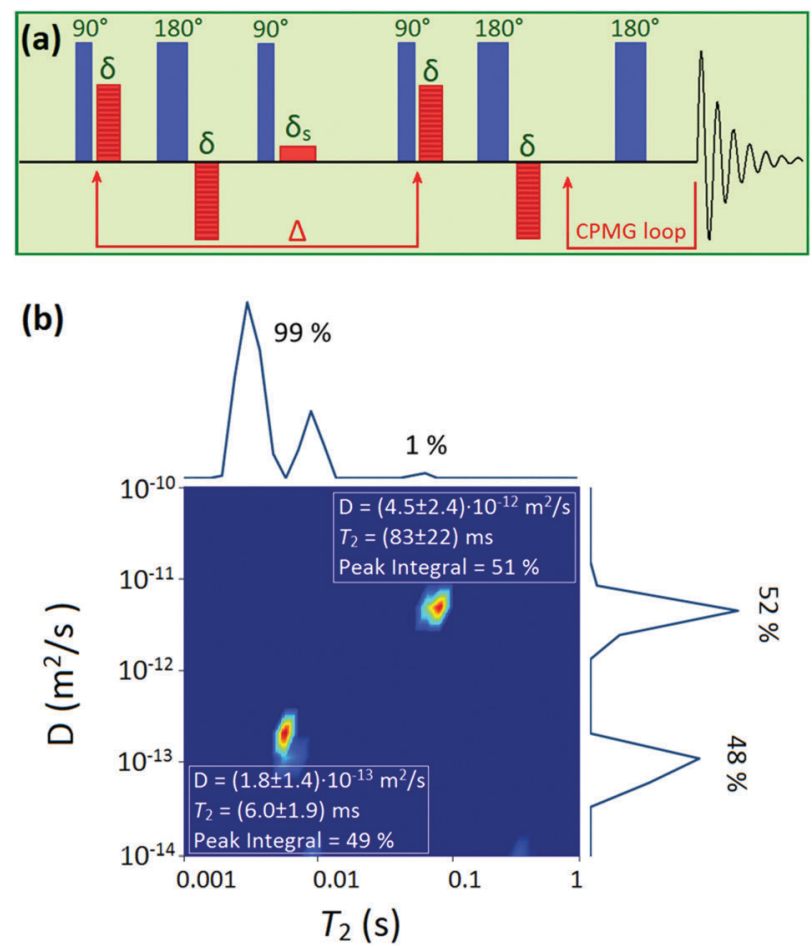

Fig. 2 (a) $D-T_{2}$ correlation pulse sequence. (b) $D-T_{2}$ relaxation correlation map for the cation at $296 \mathrm{~K}$. The experimental time was 19 hours with 32 scans. 1D $T_{2}$ relaxation time and diffusion coefficient distributions measured using the CPMG and PGSTE-BP methods, along with the relative integrals of the slow and fast components, are shown on the top and right of the map, respectively. The integrals of the peaks in the $D$ and $D-T_{2}$ distributions are heavily $T_{2}$ weighted, while the integrals of the peaks in the $T_{2}$ distribution reflect the real proportions of the phases (see text). The $T_{2}$ distributions represent the $T_{2}$ values of all the cation protons in the two phases (see text). The errors for the $D$ and $T_{2}$ values shown in the figure represent the ranges of $D$ and $T_{2}$ for each peak.

However, because the overall time the magnetisation remained in the transverse plane in the PGSTE-BP diffusion experiment and in the diffusion encoding part of the $D-T_{2}$ experiment was $22 \mathrm{~ms}$, the final magnetisation of the slow diffusing phase was less than $3 \%$ of its initial value due to $T_{2}$ relaxation, while about $76 \%$ of the magnetisation of the fast diffusing component remained. Therefore, the amplitudes in the $D-T_{2}$ map and 1D diffusion coefficient distribution do not reflect real proportions of phases; the real proportion of the slow phase is actually much higher than that of the fast phase.

Once the $T_{2}$ values of the slow and fast phases were identified using $D-T_{2}$ correlation experiments, the real proportions of the phases could be determined from the cation $T_{2}$ distributions measured using the CPMG sequence (see Fig. 2b, top). The relative integrals of the slow and fast components are $99 \%$ and $1 \%$, respectively, i.e., the vast majority of the cations (and anions) reside in the aggregated phase, and only a small fraction belong to the free, non-aggregated phase. The slow component is split into two peaks in the $T_{2}$ distribution. The component includes a range of different relaxation times of protons in the $\mathrm{P}^{-\mathrm{CH}_{2}}, \mathrm{CH}_{2}$ and $\mathrm{CH}_{3}$ groups of the cation, and the inverse Laplace transform has a tendency to split up a broad peak into a set of narrower peaks (so-called pearling artefacts). ${ }^{4 a}$ 

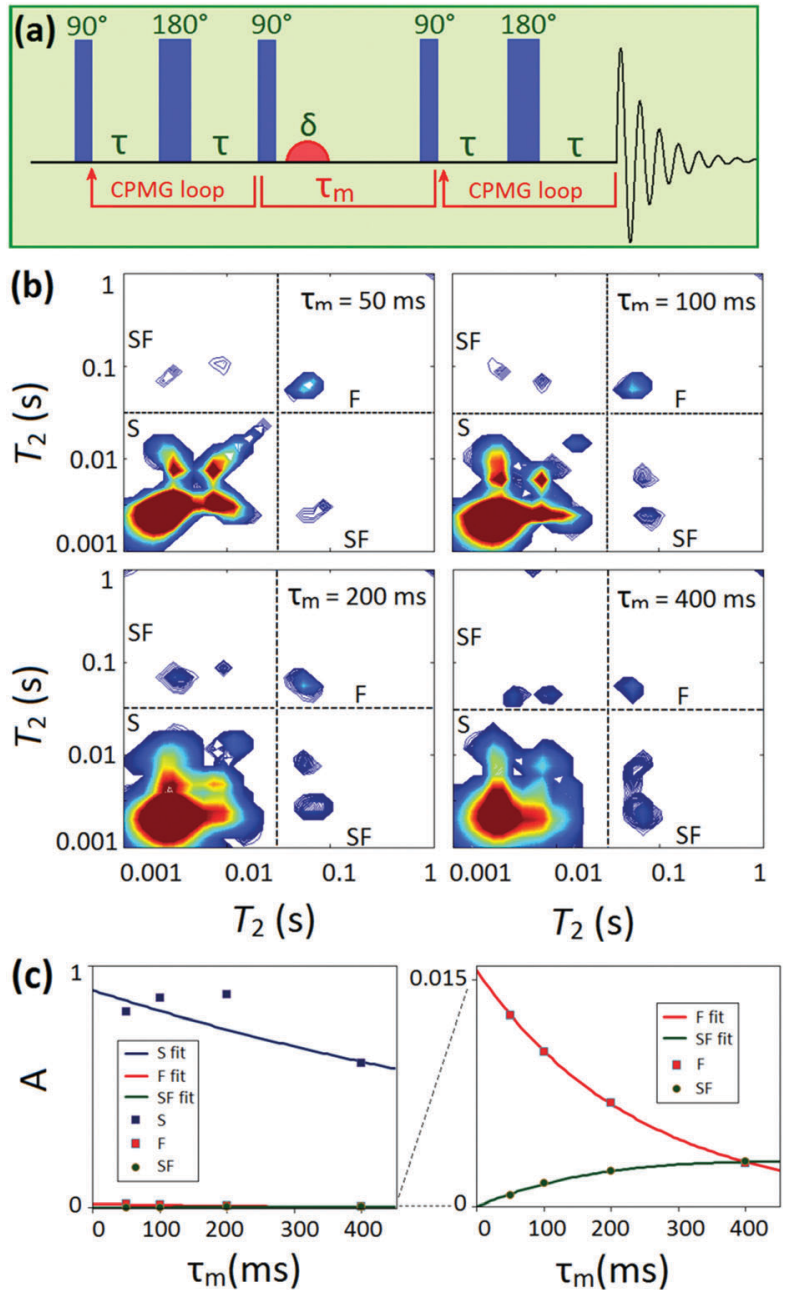

Fig. 3 (a) $T_{2}-T_{2}$ relaxation exchange pulse sequence. (b) Cation $T_{2}-T_{2}$ maps as a function of mixing time $\tau_{\mathrm{m}}$ at $296 \mathrm{~K}$. The experimental time was about $8 \mathrm{~h}$ with 8 scans. Regions of the diagonal peaks of the slow and fast components (S and F) and the cross-peaks (SF) are indicated by dashed lines. (c) Integrals of the peaks as a function of the mixing time. Fit of the two-site exchange model to the data is shown by solid lines.

We investigated the chemical exchange of the cations between the slow and fast phases at $296 \mathrm{~K}$ using the $T_{2}-T_{2}$ relaxation exchange sequence ${ }^{13}$ shown in Fig. 3a. We note that it is not possible to investigate the exchange using the traditional exchange spectroscopy (EXSY) method, ${ }^{14}$ because the exchanging sites are not resolved in the spectrum; ${ }^{1} \mathrm{H}$ chemical shifts of protons in cations and anions in the slow and fast phases are equal. The sequence includes two CPMG-type $T_{2}$ loops separated by a mixing time $\tau_{\mathrm{m}}$. The resulting $T_{2}-T_{2}$ maps, shown in Fig. $3 \mathrm{~b}$, include a set of diagonal peaks arising from the slow phase, labelled " $\mathrm{S}$ ", and a single diagonal peak from the fast phase, labelled "F". Furthermore, there are cross-peaks, labelled "SF", indicating that there is an exchange of the cations between the slow and fast phases. A fit of the two site exchange model ${ }^{14}$ to the integrals of the cross- and diagonal peaks, shown in Fig. 3c, results in a rather small exchange rate, $k=1.11 \pm 0.06 \mathrm{~s}^{-1}\left(k_{\mathrm{SF}}=0.0190 \pm 0.0009 \mathrm{~s}^{-1}\right.$, $\left.k_{\mathrm{FS}}=1.09 \pm 0.05 \mathrm{~s}^{-1}\right)$. The amplitude of the $\mathrm{S}$ peak fluctuates with the mixing time, because the signal is close to the edge of the map.
Therefore, this amplitude was fixed in the fit. The exchange rates of the anions are equal to the exchange rates of the cations within experimental errors (see Fig. S7, ESI $\dagger$ ). The "lifetimes" of ions in the slow and fast phases $\left(k_{\mathrm{SF}}{ }^{-1}\right.$ and $\left.k_{\mathrm{FS}}{ }^{-1}\right)$ are about 52 and $0.92 \mathrm{~s}$, respectively.

Relaxation modelling ${ }^{15}$ provides a means to quantitatively test the proposed hypothesis and gain more detailed understanding of the microscopic structure of the IL. The experimental relaxation data were interpreted in terms of a model of spheres (see details in the ESI $\dagger$ ). The model contains three types of spheres: larger spheres of aggregates of anions and cations, and smaller spheres of either free anions or free cations. The model includes an effective $\mathrm{H}-\mathrm{H}$ distance (intrasphere), and reorientational and translational diffusion correlation times, where the latter involve the experimentally known diffusion constants for anions or cations in the slow or fast phases. The upper boundary of possible diffusion dynamics in the model parameterization is effectively taken to be the noninteracting hard spheres. This is due to the molecular interactions expected to introduce significant deviation and slow down the diffusion for this system. The model accounts for interionic diffusion modulated dipole-dipole (inter-DD), intra-ionic dipole-dipole (intra-DD) and proton chemical shift anisotropy (CSA) relaxation mechanisms.

Altogether eight $T_{2}$ and four $T_{1}{ }^{1} \mathrm{H}$ relaxation times recorded at 300 and $600 \mathrm{MHz}$ were analysed. The data supports a model where a larger aggregate rotates slowly and free ions (or smaller aggregates) rotate more rapidly with rotational correlation times of $7 \mu \mathrm{s}$ and $10 \mathrm{~ns}$, and diameters of 50-160 and 15-30 A, respectively. The latter diameter roughly matches with the diameters of the cation and anion. Markov-chain Monte Carlo simulation was performed to estimate $95 \%$ confidence intervals. The best-fit model was found for a spin fraction $99.5 \%$ in the slowly diffusing aggregate, which is close to the experimentally determined fraction (99\%). The dominating mechanisms for fast and slowly diffusing aggregates are inter-DD and intra-DD, respectively, but CSA also has a non-negligible role. The volume ratio suggests that the aggregates are formed by 10-70 ion pairs, meaning that there are 1-10 aggregates per free anion-cation pair. Considering the free energy of the system, these aggregates are expected to be neutral or of lower net charge.

Self-aggregation (micellisation) of ILs has been earlier observed in aqueous solutions. ${ }^{16}$ The mechanism of the hypothesised aggregation cannot be the same in the $\left[\mathrm{P}_{6,6,6,14}\right][\mathrm{BMB}] \mathrm{IL}$, because the residual water was removed from the IL with a vacuum treatment at $383 \mathrm{~K}$ (see details in the ESI $\dagger$ ). The aggregation could be induced by van der Waals interactions between the alkyl chains of cations and the aromatic groups of anions, tail group aggregation $^{17}$ or impurities. The identification of the mechanism of proposed aggregate formation in $\left[\mathrm{P}_{6,6,6,14}\right][\mathrm{BMB}]$ is outside the scope of the current work.

In the 1D diffusion distributions shown in Fig. 1c and d, the peak of the fast diffusing phase disappears at higher temperatures. It is counterintuitive to assume that free ions (or smaller aggregates) exist only at lower temperatures (i.e., below $320 \mathrm{~K}$ ). Therefore, we expect that the reason for the disappearance is 
the increased exchange rate between the low and fast phases with temperature. Significantly narrowed line-widths in ${ }^{1} \mathrm{H}$ NMR spectra measured at higher temperatures also indicate the faster mobility of the ions (compare spectra in Fig. 1a and b). Due to the fast exchange, the two peaks in the diffusion distributions merge into one. Because the population of the ions in the slow phase is much higher than in the fast phase, the merged peak resides close to the $D$ of the slow phase. The activation energies of diffusion are shown in Fig. S3 (ESI $\dagger$ ).

The NMR chemical shift of ${ }^{129} \mathrm{Xe}$ is extremely sensitive to its local environment, and therefore Xe is a very useful inert probe in materials research and medical and biochemical applications. ${ }^{18}$ Recently, ${ }^{129}$ Xe NMR has also been exploited in IL research. ${ }^{19}$ Typically, the phase change of liquid or liquid crystals is manifested by an abrupt change in the chemical shift of xenon dissolved in the liquid. ${ }^{20}$ The linear decrease of the ${ }^{129}$ Xe chemical shift of xenon dissolved in the $\left[\mathrm{P}_{6,6,6,14}\right][\mathrm{BMB}] \mathrm{IL}$ with temperature indicates that there are no abrupt changes in the density and orientational order parameter of the IL in the $294-373 \mathrm{~K}$ temperature range (see Fig. S6, ESI $\dagger)^{20}{ }^{20}$ This indicates that there are no phase transitions within that temperature interval. Furthermore, the observation supports the abovementioned interpretation that the disappearance of the fast diffusion component at higher temperatures cannot be associated with such a change.

In summary, we have demonstrated that multidimensional LNMR provides detailed information about the structure and dynamics of ILs that is not possible to obtain using other methods. It clearly reveals intriguing two coexisting dynamic phases in the $\left[\mathrm{P}_{6,6,6,14}\right][\mathrm{BMB}] \mathrm{IL}$, and it allows the estimation of the size of the hypothesised IL aggregates and the exchange rate between the aggregates and free ions (or smaller aggregates). These observations pave the way for better understanding of useful properties of ILs and designing new task specific ILs. In the future, the sensitivity and time-efficiency of the LNMR method can be improved by several orders of magnitude using hyperpolarised ultrafast LNMR approaches, ${ }^{21}$ which enable monitoring fast processes, such as chemical reactions and phase changes, in ILs in real time.

V.-V. T. acknowledges the Academy of Finland for financial support (grant numbers 289649 and 294027). P. H. acknowledges the grant for the EU Horizon 2020 research and innovation programme under the Marie Skłodowska-Curie grant number 703446. The Knut and Alice Wallenberg foundation (project number KAW 2012.0078 (S. G., O. N. A., F. U. S. and A. F.)) and the Swedish Research Council (project numbers 621-20135171 (O. N. A. and F. U. S.), 621-2011-4600 and 621-2014-4694 (S. G. and O. N. A.)) are gratefully acknowledged for their financial support. The Laplace inversion programme was provided by the late Prof. P. Callaghan.

\section{Conflicts of interest}

There are no conflicts to declare.

\section{References}

1 M. Freemantle, An introduction to ionic liquids, Royal Society of Chemistry, Cambridge, 2009.

2 P. T. Callaghan, Translational Dynamics and Magnetic Resonance: Principles of Pulsed Gradient Spin Echo NMR, Oxford University Press, Oxford, 2011.

3 D. E. Freed, L. Burcaw and Y.-Q. Song, Phys. Rev. Lett., 2005, 94, 067602.

4 (a) D. Bernin and D. Topgaard, Curr. Opin. Colloid Interface Sci., 2013, 18, 166-172; (b) Y. Q. Song, J. Magn. Reson., 2013, 229, 12-24.

5 (a) L. Venkataramanan, Y.-Q. Song and M. D. Hürlimann, IEEE Trans. Acoust., Speech, Signal Process., 2002, 50, 1017-1026; (b) Y.-Q. Song, L. Venkataramanan, M. D. Hürlimann, M. Flaum, P. Frulla and C. Straley, J. Magn. Reson., 2002, 154, 261-268.

6 K. Damodaran, Annu. Rep. NMR Spectrosc., 2016, 88, 215-244.

7 (a) F. U. Shah, S. Glavatskih, D. R. MacFarlane, A. Somers, M. Forsyth and O. N. Antzutkin, Phys. Chem. Chem. Phys., 2011, 13, 12865-12873; (b) F. U. Shah, S. Glavatskih, P. M. Dean, D. R. MacFarlane, M. Forsyth and O. N. Antzutkin, J. Mater. Chem., 2012, 22, 6928-6938.

8 A. Filippov, F. U. Shah, M. Taher, S. Glavatskih and O. N. Antzutkin, Phys. Chem. Chem. Phys., 2013, 15, 9281-9287.

9 R. M. Cotts, M. J. R. Hoch, T. Sun and J. T. Marker, J. Magn. Reson., 1989, 83, 252-266.

10 S. Godefroy and P. T. Callaghan, Magn. Reson. Imaging, 2003, 21, 381-383.

11 M. D. Hürlimann and L. Venkataramanan, J. Magn. Reson., 2002, 157, 31-42.

12 S. Meiboom and D. Gill, Rev. Sci. Instrum., 1958, 29, 688-691.

13 J.-H. Lee, C. Labadie, C. S. Springer, Jr. and G. S. Harbison, J. Am. Chem. Soc., 1993, 115, 7761-7764.

14 J. Jeener, B. H. Meier, P. Bachmann and R. R. Ernst, J. Chem. Phys., 1979, 71, 4546-4553.

15 (a) Y. Ayant, E. Belorizky, J. Alizon and J. Gallice, J. Phys., 1975, 36, 991-1004; (b) L. P. Hwang and J. H. Freed, J. Chem. Phys., 1975, 63, 4017-4025; (c) D. Kruk, R. Meier, A. Rachocki, A. Korpała, R. K. Singh and E. A. Rössler, J. Chem. Phys., 2014, 140, 244509; (d) P.-O. Westlund, G. Driver, Y. Wang, T. Sparrman, A. Laaksonen and Y. Huang, Phys. Chem. Chem. Phys., 2017, 19, 4975-4988.

16 (a) M. Figueira-Gonzalez, V. Francisco, L. García-Río, E. F. Marques, M. Parajo and P. Rodríguez-Dafonte, J. Phys. Chem. B, 2013, 117, 2926-2937; (b) V. Klimavicius, V. Bacevicius, Z. Gdaniec and V. Balevicius, J. Mol. Liq., 2015, 210, 223-226.

17 Y. Wang and G. A. Voth, J. Am. Chem. Soc., 2005, 127, 12192-12193.

18 D. A. Barskiy, A. M. Coffey, P. Nikolaou, D. M. Mikhaylov, B. M. Goodson, R. T. Branca, G. J. Lu, M. G. Shapiro, V.-V. Telkki, V. V. Zhivonitko, I. V. Koptyug, O. G. Salnikov, K. V. Kovtunov, V. I. Bukhtiyarov, M. S. Rosen, M. J. Barlow, S. Safavi, I. P. Hall, L. Schröder and E. Y. Chekmenev, Chem. - Eur. J., 2017, 23, 725-751.

19 (a) F. Castiglione, R. Simonutti, M. Mauri and A. Mele, J. Phys. Chem. Lett., 2013, 4, 1609-1612; (b) P. Morgado, K. Shimizu, J. M. S. S. Esperanc, P. M. Reis, L. P. N. Rebelo, J. N. Canongia Lopes and E. J. M. Filipe, J. Phys. Chem. Lett., 2013, 4, 2758-2762; (c) G. Saielli, A. Bagno, F. Castiglione, R. Simonutti, M. Mauri and A. Mele, J. Phys. Chem. B, 2014, 118, 13963-13968.

20 M. Ylihautala, J. Lounila and J. Jokisaari, J. Chem. Phys., 1999, 110(13), 6381-6388.

21 (a) S. Ahola, V. V. Zhivonitko, O. Mankinen, G. Zhang, A. M. Kantola, H.-Y. Chen, C. Hilty, I. V. Koptyug and V.-V. Telkki, Nat. Commun., 2015, 6, 8363; (b) S. Ahola and V.-V. Telkki, ChemPhysChem, 2014, 15, 1687-1692; (c) J. N. King, V. J. Lee, S. Ahola, V.-V. Telkki and T. Meldrum, Angew. Chem., Int. Ed., 2016, 55, 5040-5043. 